\title{
Effect of acylation on the antimicrobial activity of temporin B analogues
}

Concetta Avitabile ${ }^{1}$, Luca Domenico D'Andrea ${ }^{1}$, Elisabetta D'Aversa ${ }^{2}$, Roberta Milani ${ }^{2}$, Roberto Gambari ${ }^{2}$, Alessandra Romanelli ${ }^{3}$

1: Institute of Biostructure and Bioimaging, CNR, via Mezzocannone 16, 80134 NaplesItaly

2: Department of Life Sciences and Biotechnology, University of Ferrara, via Fossato di Mortara 74, 44121- Italy

3: University of Milan, Department of Pharmaceutical Sciences, via Venezian 21, 20133 Milano

Corresponding author e-mail: alessandra.romanelli@unimi.it

\begin{abstract}
New peptides derived from the natural antimicrobial temporin B were obtained. The design, the antimicrobial activity as well as the characterization of the secondary structure of peptides in the presence of bacterial cells is here described. We identified position 6 of temporin $B$ as the most suitable for the introduction of acyl chains and we identified TB_KKG6K as the most active analogue against Gram positive and Gram negative bacteria as compared to natural temporin $B$ and to TB_KKG6A.
\end{abstract}

\section{Introduction}

Natural antimicrobial peptides are largely investigated due to their broad spectrum of action and to their scarce ability to induce resistance in bacterial cells. They are interesting scaffolds for the development of new and more potent antibiotics. Conjugation of peptides to lipid chains has been demonstrated as a useful strategy to improve and expand the activity spectrum of these compounds. Many examples are reported in the literature, showing how, generally, conjugation at the $\mathrm{N}$ or $\mathrm{C}$ terminus of a peptide with a lipid chain results in an enhancement of its biological activity. Conjugation at the C-terminus of the peptide lactoferricin by a $\mathrm{C} 12$ acyl chain enhances its antimicrobial activity as well as its affinity for lipid $A{ }^{[1]}$ Modification of the ultrashort peptide OOWW by lauric acid at the $\mathrm{N}$ terminus results in an increased antimicrobial effect against planktonic and biofilm forms of Gram positive microorganisms. ${ }^{[2]}$ Recent studies carried out on beta defensin analogues demonstrated that $\mathrm{N}$-terminal acylation by a C8-C10 chain positively affects the antimicrobial activity of compounds: modified peptides show rapid killing kinetics and synergism with conventional antibiotics. ${ }^{[3]}$ It has been hypothesized that lipidated peptides act by targeting the bacterial plasma membrane, as well as some natural lipidated peptides ${ }^{[4][5]}$.

With the aim to obtain potent antimicrobial peptides, we designed a series of peptides analogues of temporin B. We had previously demonstrated that addition of two extra 
lysines at the $\mathrm{N}$-terminus ${ }^{[6]}$ and substitution of the glycine 6 residue by an alanine in temporin $B$ results in a peptide, named TB_KKG6A (sequence: KKLLPIVKNLLKSLL), showing activity at low concentrations against Gram positive and Gram negative bacteria, while the natural temporin $B$ is only active against Gram positive bacteria. ${ }^{[7]}$ The threedimensional structure of the peptide obtained by NMR either in the presence of E.coli LPS and in the presence of E.coli cells shows that the peptide assumes an $\alpha$-helical conformation. ${ }^{[7 a, 8]}$ NMR paramagnetic relaxation experiments, carried out to detect the orientation of the peptide with respect to LPS revealed that side chains of Pro3, Ala 6, Asn 7, Leu12 and 13 penetrated the LPS. The side chain of Lys10 was found in two different orientations: either on the surface and inside the LPS. Saturation transfer difference experiments carried out on the peptide TB_KKG6A in the presence of E.coli cells confirmed that residues 3,6 and 10 are in close contact with bacterial cells ${ }^{[8]}$.

We hypothesized that the replacement of amino acids in positions which seem to be involved hydrophobic interactions with the LPS by acylated residues may result in an increase of their antimicrobial activity. For these reason we synthesized a number of temporin $B$ analogues, and tested the effect of the position and length of the acyl chain on the antimicrobial activity of the peptides. Secondary structure of the peptides in the presence of Gram positive and Gram negative bacterial cells was also investigated.

\section{Results}

2.1 Design and antimicrobial activity .

Based on the results of NMR experiments of the peptide TB_KKG6A in the presence of E.coli LPS and cells, we designed an initial set of molecules (peptides 1 to 6), in which Pro3, Gly6 and Asn7, found to penetrate LPS micelles, were replaced by acylated lysine residues (Table 1 ).

Table 1. Peptide sequences, names and numbers

\begin{tabular}{|l|l|l|}
\hline Peptide number & Peptide sequence $^{1}$ & Name $^{1}$ \\
\hline 1 & KKLLK(Pal)IVANLLKSLL & TB_KKP3K(Pal)G6A \\
\hline 2 & KKLL K(Oct)IVANLLKSLL & TB_KKP3K(Oct)G6A \\
\hline 3 & KKLLPIV K(Pal)NLLKSLL & TB_KKG6K(Pal) \\
\hline 4 & KKLLPIV K(Oct)NLLKSLL & TB_KKG6K(Oct) \\
\hline 5 & KKLLPIVA K(Pal)LLKSLL & TB_KKG6AN7K(Pal) \\
\hline 6 & KKLLPIVA K(Oct)LLKSLL & TB_KKG6AN7K(Oct) \\
\hline 7 & KKLLPIVKNLLKSLL & TB_KKG6K \\
\hline 8 & KKLLPIVK(Ac)NLLKSLL & TB_KKG6K(Ac) \\
\hline 9 & KKLLPIVK(DAVA)NLLKSLL & TB_KKG6K(DAVA) \\
\hline 10 & KKLLPIVK(Ahx)NLLKSLL & TB_KKG6K(Ahx) \\
\hline 11 & KKLLPIVK(BrHx)NLLKSLL & TB_KKG6K(BrHx) \\
\hline 12 & KKLLPIVK(Aund)NLLKSLL & TB_KKG6K(Aund) \\
\hline 1
\end{tabular}

${ }^{1} \mathrm{BrHx}$ : 6-bromo hexanoic acid; Aund: 11-amino undecanoic acid

These experiments were aimed at identifying the position in which the acylation produced beneficial effects on the antimicrobial activity. Residues in position 10, 12 and 13, that are also included between those involved in the penetration of LPS, were not modified as these were demonstrated to be crucial to maintain the activity of the temporin $B$ peptide. ${ }^{[7 a]}$ To start, we investigated killing kinetics for peptides 1 to 6 , bearing in positions 3 or 6 or 7 
lysines with chains of two different length, octanoic acid and palmitic acid respectively on two different bacteria, E.coli and S.epidermidis (Figure 1).

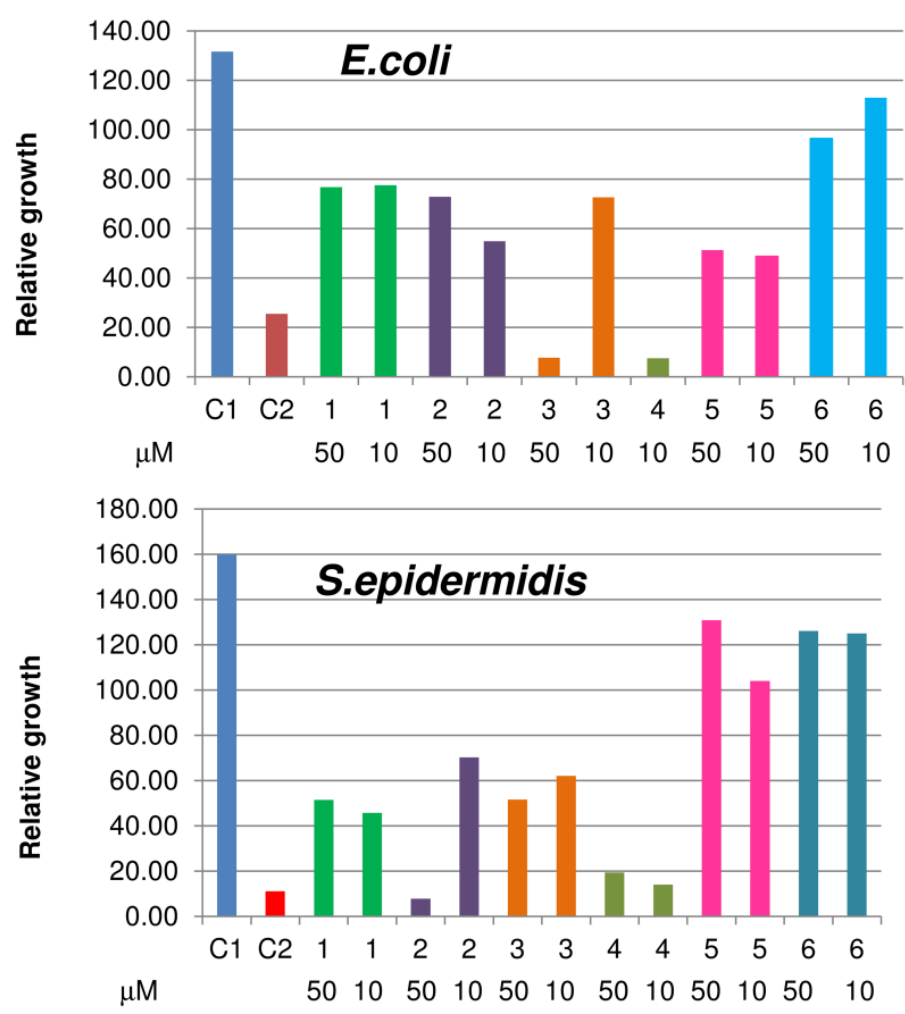

Figure 1. Antimicrobial activity of palmitoylated and octanoylated peptides. Effect of 24 hours treatment of bacterial cells with indicated peptides at two different concentrations. Relative growth of bacteria was calculated as the ratio of the $\mathrm{OD}_{600}$ after 24 hours incubation of peptides with cells and at time zero. C1: control cells, C2: gentamicin, used at $1.5 \mu \mathrm{g} / \mathrm{mL}$ for E.coli cells and at $1 \mu \mathrm{g} / \mathrm{ml}$ for S.epidermidis cells

Replacement of amino acids in position 3 and 7 by acylated lysines yielded peptides with a very low antimicrobial activity against E.coli. Replacement of proline 3 by octanoylated lysine yielded peptide n.2; antimicrobial activity against S.epidermidis required a concentration of $50 \mu \mathrm{M}$. Replacement of glycine 6 by acylated lysines resulted in promising analogues (Figure 1 and 2): the octanoylated peptide (peptide n.4) showed very strong activity either against E.coli and S.epidermidis at the lowest concentration tested and fast killing. The palmitoylated peptide (peptide number 3) was active against E.coli only at high concentration, while the activity against S.epidermidis was modest at both concentrations tested. Based on these results we decided to investigate the effect of introduction of acyl chains of different length in position 6. We synthesized new analogues, with lysine in position 6 (TB_KKG6K) and with lysine acetylated (TB_KKG6K(Ac)), acylated with 5amino valeric acid (TB_KKG6K(DAVA)), 6-amino hexanoic acid (TB_KKG6K(Ahx)), 6bromo hexanoic acid (TB_KKG6K(BrHx)) and 11-amino undecanoic acid (TB_KKG6K(Aund)) (Table 1). MIC for these new set of peptides were determined against E.coli, S.epidermidis and P.aeruginosa. Peptides with short acyl chains resulted more active than peptides with longer acyl chains, with TB_KKG6K being the most active 
peptide. All peptides show an increased antimicrobial activity as compared to the parent TB_KKG6A against P.aeruginosa, with the exception of TB_KKG6K(DAVA) (Table 2).

Table 2. Antimicrobial activity of selected peptides

\begin{tabular}{|l|l|l|l|}
\hline \multirow{2}{*}{$\begin{array}{l}\text { Peptide } \\
\text { number }\end{array}$} & \multicolumn{3}{|c|}{ MIC $(\mu \mathrm{M})$} \\
\cline { 2 - 4 } & E. coli & S. epidermidis & P. aeruginosa \\
\hline 7 & 0.2 & 0.025 & 2.5 \\
\hline 8 & 0.5 & 0.1 & 2.5 \\
\hline 9 & 1.25 & 0.1 & 15 \\
\hline 10 & 1.65 & 0.25 & 7 \\
\hline 11 & 1 & 2.5 & 5 \\
\hline 12 & 5 & 2.5 & 3 \\
\hline
\end{tabular}
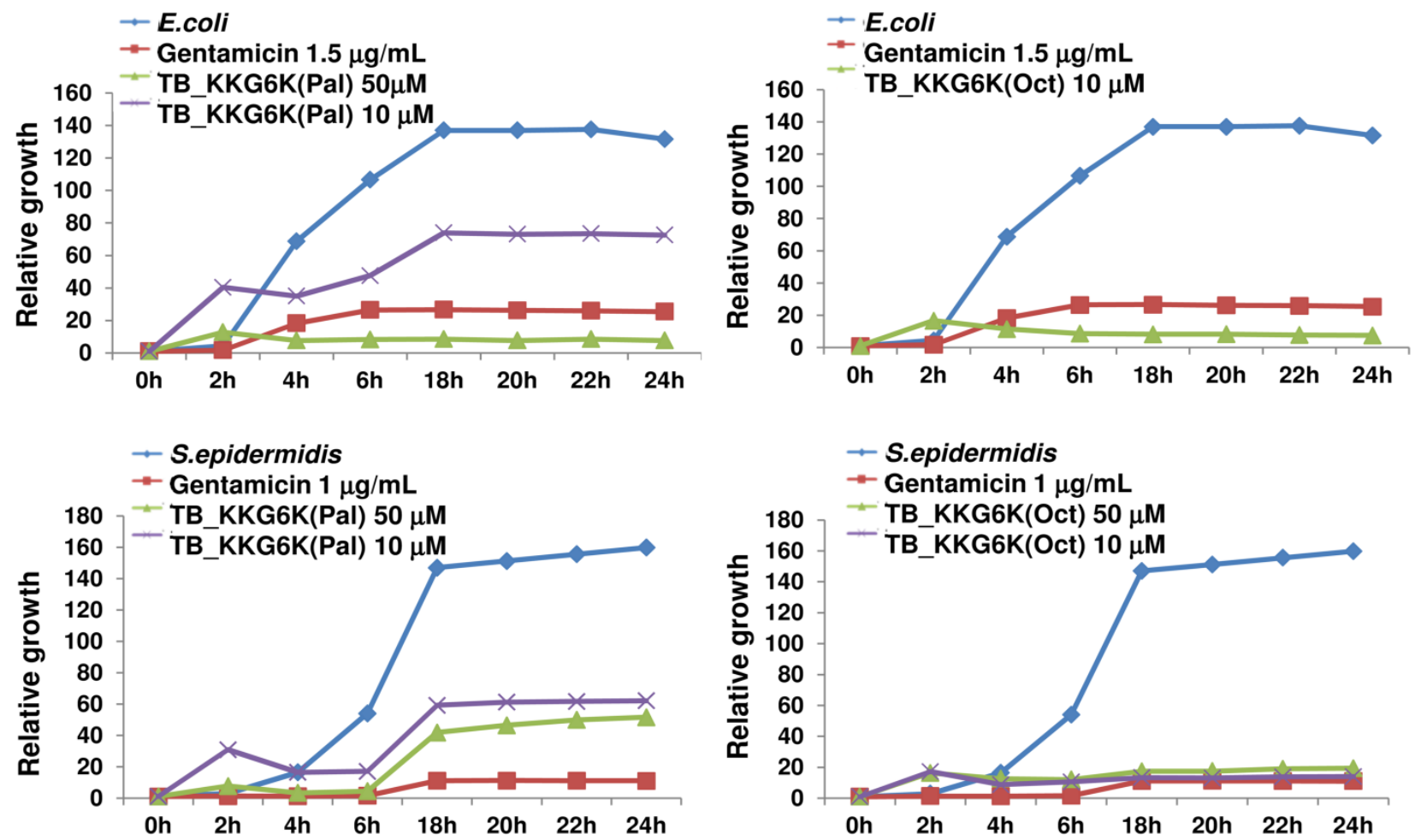

Figure 2: Killing kinetics of peptides modified in position 6. Relative growth of bacteria was calculated as the ratio of the $\mathrm{OD}_{600}$ after the indicated time of incubation of peptides with cells and at time zero.

\subsection{CD studies}

The secondary structure of the most active peptide TB_KKG6K was investigated in phosphate buffer and in the presence of S.epidermidis and E.coli bacterial cells (Figure 3). TB_KKG6K is a random coil in phosphate buffer and in the presence of S.epidermidis cells. In the presence of E.coli cells, with increasing the incubation time, the negative 
maximum appearing at $200 \mathrm{~nm}$ in buffer shifts to $205 \mathrm{~nm}$ and a weak band around $222 \mathrm{~nm}$ appears. We also investigated the secondary structure of TB_KKG6K(Pal) and TB_KKG6K(Oct) in the same experimental conditions, with the aim to understand the effect of lipidation on the structure of the peptides (Figure 3). The peptide TB_KKG6K(Pal) is an $\alpha$-helix in phosphate buffer; upon interaction with E.coli and S.epidermidis cells signals become less intense and noisy. TB_KKG6K(Oct) assumes an $\alpha$-helical structure in phosphate buffer $;{ }^{[9]}$ when the peptide is incubated with E.coli and S.epidermidis cells, CD spectra recorded at different time of incubation show one negative maximum around 200 $\mathrm{nm}$.
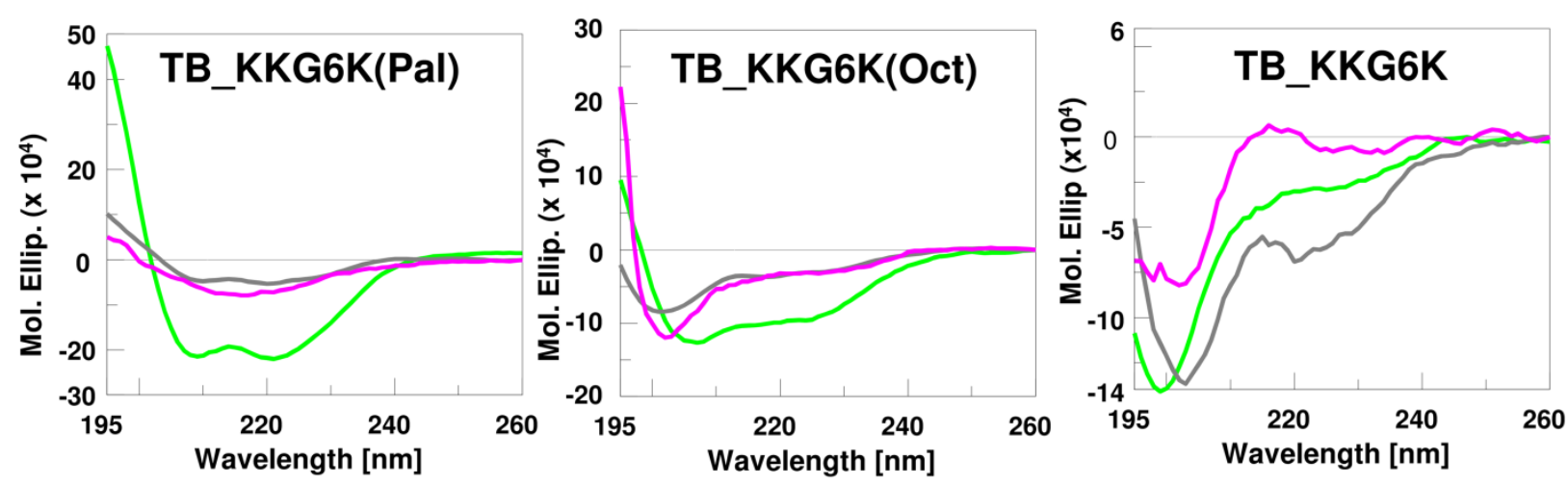

Figure 3: $\mathrm{CD}$ spectra of peptides in phosphate buffer, $\mathrm{pH} 7$ (green), in the presence of E.coli cells (grey) or S.epidermidis cells (magenta).

\subsection{Critical aggregation concentration}

The critical aggregation concentration was determined for the peptide TB_KKG6K(Pal) and TB_KKG6K by fluorescence experiments, using as a probe 8-amino naphtalen sulfonic acid. This probe emits between 460 and $480 \mathrm{~nm}$ if it is in a hydrophobic environment. Emission at $470 \mathrm{~nm}$ was plotted against peptide concentration (Figure 4). The critical aggregation concentration was evaluated to be $0.26 \mu \mathrm{M}$ for TB_KKG6K(Pal). TB_KKG6K does not aggregate up to $10 \mathrm{mM}$ concentration.

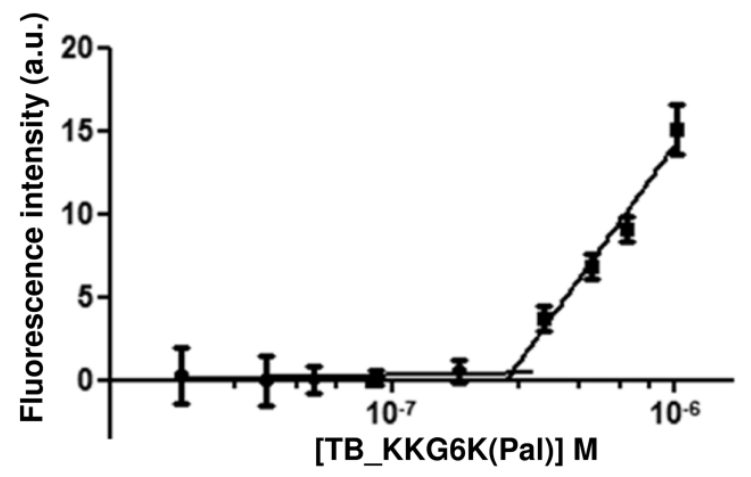


Figure 4: Critical aggregation concentration plot for the peptide TB_KKG6K(Pal).

\section{Discussion}

The antimicrobial activity of peptides is related to their ability to bind to and disrupt bacterial membranes. Electrostatic interactions between the positively charged residues of antimicrobial peptides and negatively charged lipopolysaccharide, in case of Gram negative bacteria, or teichoic acids, in case of Gram positive bacteria, are responsible for the initial interaction of peptides with bacterial cells. Hydrophobic residues instead seem to penetrate bacterial membrane, to interact with lipids. Some peptides assume an amphipathic structure, that grants a clear separation between the hydrophobic and hydrophilic patches, ${ }^{[10]}$ maximizing the interactions between peptides and bacterial membranes. TB_KKG6A is not an amphipathic peptide; nevertheless it is highly active against Gram positive and Gram negative bacteria. It assumes an $\alpha$-helical structure in the presence of E.coli cells, while it does not show a preferred secondary structure in the presence of S.epidermidis cells. ${ }^{[11]}$ Binding of the peptide to E.coli cells causes aggregation and death of bacterial cells ${ }^{[2]}$. With the aim to increase the antimicrobial activity of this peptide by reinforcing the hydrophobic interactions of the peptide with bacterial cell membrane, we investigated the effect of introduction of acyl chains of different length in different positions of the peptides, chosen between those hypothesized to penetrate the membrane and not essential for the activity of the peptides, i.e. positions 3, 6 and 7. The initial screening, carried out replacing Pro3, Ala 6 and Asn 7 of TB_KKG6A with either $\mathrm{Lys}(\mathrm{Pal})$ and Lys(Oct), revealed that modifications in position 6 are beneficial for the activity of the peptide. These results are in accordance with those reported on TB analogues obtained by Ala scan experiments, showing that TB_P3A, and TB_N7A were less active than TB_G6A. ${ }^{[7 a]}$ Peptides modified by the long palmitic acid chain in position 6 are less active as compared to peptides modified with shorter acyl chains.

Based on the results of the initial screening we synthesized a new set of analogues modified in position 6 by a lysine or an acylated lysine. The most active peptide is TB_KKG6K; introduction of a lysine residue with its short acyl side chain and its positive charge increases the antimicrobial activity against Gram positive and Gram negative bacteria likely because interactions with negatively charged outer membranes are favored. The 4 carbon atom chain is likely able to destabilize bacterial membrane, and too short to promote aggregation of the peptide. Acetylation of the lysine residue in position 6 reduces the activity of the peptide against E.coli and S.epidermidis; the effect of acetylation is double: suppression of the positive charge and tiny increase of the acyl chain length. When the positive charge is kept fixed at 4 and the acyl chain length is increased, as in TB_KKG6K(DAVA), TB_KKG6K(Ahx), TB_KKG6K(Aund) we observe a decrease of the antimicrobial activity of the peptides against E.coli and S.epidermidis, suggesting that the increase of the hydrophobicity of the peptide is detrimental to the antimicrobial activity. Effects of the hydrophobicity on the antimicrobial activity are remarkable for the Gram positive bacterium S.epidermidis; the comparison of the activity of the analogs TB_KKG6K(Ahx) and TB_KKG6K(BrHx) is consistent with this observation. The activity of 
the peptides was also tested against $P$.aeruginosa, a Gram negative bacterium. Results in this case are difficult to interpret as there is no apparent relationship between the hydrophobicity of the peptides and their activity. Importantly, with the exception of TB_KKG6K(DAVA), all peptides are more active than TB_KKG6A against $P$.aeruginosa.

The presence of acyl chains affects the structure of the peptides. CD reveals that TB_KKG6K(Pal) possess an $\alpha$-helical structure in phosphate buffer, that disappears in the presence of bacterial cells. The palmitoylated peptide show a strong tendence to aggregate; in fact, the critical aggregation concentration calculated for the peptide TB_KKG6K(Pal) is very low, $0.26 \mu \mathrm{M}$. As reported in the literature, loss of conformational freedom due to tethering and confinement leads to folding into defined secondary structure motifs. ${ }^{[13]}$ This effect has been reported for other palmitoylated peptide and is likely what we are observing here for the palmitoylated peptide. ${ }^{[14]}$ Interactions of TB_KKG6K(Pal) aggregates with bacterial cells, either E.coli and S.epidermidis, likely cause disgregation of the aggregates that results in a decrease in the intensity of CD spectra in the presence of E.coli cells, and also in a change in shape of the spectra in the case of S. epidermidis cells. Indeed the free TB_KKG6K(Pal) peptide does not induce massive bacterial cell death. Similar results have been obtained also for the peptide TB_KKG6K(Oct). TB_KKG6K is unfolded either in buffer and in the presence of S.epidermidis bacterial cells, while some degree of secondary structure appears in the presence of E.coli cells. The ability to fold upon interaction with E.coli is associated in many cases with the antimicrobial activity of the peptide, and this is consistent with results reported for other natural peptides, such as temporin $L$, cecropin $A$ and magainin 2 or obtained upon modification of temporin peptides such as TB_KKG6A and LG21. ${ }^{[8,11,15]}$

\section{Conclusions}

We obtained a new set of analogues of the peptide temporin B, showing activity either against Gram positive and against Gram negative bacteria. Introduction of short the acyl chains in position 6 as well as of lysines at the $\mathrm{N}$-terminus of temporin B positively affects the antimicrobial activity of the peptides against E.coli and S.epidermidis. Replacement of glycine 6 by a lysine results in the most active peptide. The length of the chain in position 6 seems to be crucial to determine the antimicrobial activity against S.epidermidis.

\section{Experimental procedures}

\subsection{Peptide synthesis}

Peptides were obtained by solid phase synthesis using standard protocols. ${ }^{[\mathrm{b}, 16]}$ FmocLys(Pal)-OH and Fmoc-Lys(Oct)-OH were used for the synthesis of palmitoylated and octanoylated peptides; Fmoc 5-amino valeric acid, Fmoc 6-amino hexanoic acid, 6 bromo hexanoic acid, Fmoc 11-aminoundecanoic acid were employed for the synthesis of the following peptides: TB_KKG6K(DAVA), TB_KKG6K(Ahx), TB_KKG6K(BrHx), TB_KKG6K(Aund). Peptide TB_KKG6K(Ac), was obtained starting from the peptide 
anchored to the resin bearing in position 6 a lysine protected with Mtt on the side chain and protected at the N-terminus with the Fmoc group. The Mtt was removed by consecutive treatments of the resin with a solution of TFA (0.1\%)/TIS (5\%) in DCM; upon neutralization the peptide was acetylated following standard protocol for peptide solid phase acetylation. ${ }^{[17]}$

Peptides were purified by RP-HPLC using a gradient of acetonitrile (0.1\% TFA) in water (0.1\% TFA) from 30 to $80 \%$ in 20 minutes (the gradient starts after 3 minutes of equilibration in $30 \%$ acetonitrile) on a Phenomenex Jupiter $10 \mu$ Proteo $90 \AA(250 \times 10 \mathrm{~mm})$ column. LC-MS analysis was performed on a by LC-MS on a LC-MS Agilent Technologies 6230 ESI-TOF using a Phenomenex Jupiter $3 \mu \mathrm{m}$ C18 $(150 \times 2.0 \mathrm{~mm})$ column with a flow rate of $0.2 \mathrm{~mL} / \mathrm{min}$. Peptides were obtained with a purity $>95 \%$, yields were calculated based on the amount of pure peptide obtained after purification.

\section{n.1: TB_KKP3K(Pal)G6A. Sequence: KKLLK(Pal)IVANLLKSLL}

Calculated mass (Da): 1931.87 found: $1931.01[\mathrm{M}+2 \mathrm{H}]^{2+}=966.50$; $[\mathrm{M}+3 \mathrm{H}]^{3+}=644.67$; r.t.: $21.1 \mathrm{~min}$, yield: $30 \%$.

n.2: TB_KKP3K(Oct)G6A. Sequence: KKLLK(Oct)IVANLLKSLL

Calculated mass (Da): 1819.64 ; found: $1820.15[\mathrm{M}+2 \mathrm{H}]^{2+}=911.29$; $[\mathrm{M}+3 \mathrm{H}]^{3+}=607.83$; r.t.: 14.7 min; yield: $25 \%$.

n.3: TB_KKG6K(Pal) Sequence: KKLLPIVK(Pal)NLLKSLL

Calculated mass $(\mathrm{Da}): 1958.85 \mathrm{~g} / \mathrm{mol}[\mathrm{M}+2 \mathrm{H}]^{2+}=980.42 ;[\mathrm{M}+3 \mathrm{H}]^{3+}=653.95$; found: $1958.23[\mathrm{M}+2 \mathrm{H}]^{2+}=980.11 ;[\mathrm{M}+3 \mathrm{H}]^{3+}=653.74 ;$ r.t.: 22.3 min; yield: $32 \%$

n.4: TB_KKG6K(Oct) Sequence: KKLLPIVK(Oct)NLLKSLL

Calculated mass (Da): 1846.62 found: $1847.01[\mathrm{M}+2 \mathrm{H}]^{2+}=924.50$; $[\mathrm{M}+3 \mathrm{H}]^{3+}=616.67$; r.t.: 16.7, yield $40 \%$

n.5: TB_KKG6AN7K(Pal) Sequence: KKLLPIVAK(Pal)LLKSLL

Calculated mass (Da): 1915.90 found: $1915.45[\mathrm{M}+2 \mathrm{H}]^{2+}=958.72$; $[\mathrm{M}+3 \mathrm{H}]^{3+}=639.48$; r.t.: 22.7 min; yield: $20 \%$

n.6: TB_KKG6AN7K(Oct) Sequence: KKLLPIVAK(Oct)LLKSLL

Calculated mass (Da): 1803,62 found: $1803,34[\mathrm{M}+2 \mathrm{H}]^{2+}=902.67$; $[\mathrm{M}+3 \mathrm{H}]^{3+}=602.11$; r.t.:

17.4 min; yield: $20 \%$

n.7:TB_KKG6K Sequence: KKLLPIVKNLLKSLL

Calculated mass (Da): 1719.30 ; found: $1719.22[\mathrm{M}+2 \mathrm{H}]^{2+}=860.62 ;[\mathrm{M}+3 \mathrm{H}]^{3+}=574.08$; r.t.:

$11.8 \mathrm{~min} ;$ yield:50\%

n.8: TB_KKG6K(Ac) Sequence: KKLLPIVK(Ac)NLLKSLL

Calculated mass (Da) 1761.30; found: $1761.26[\mathrm{M}+2 \mathrm{H}]^{2+}=881.62 ;[\mathrm{M}+3 \mathrm{H}]^{3+}=588.08$; r.t.:12.1 min; yield: $50 \%$

n.9: TB_KKG6K(DAVA) Sequence: KKLLPIVK(DAVA)NLLKSLL

Calculated mass (Da) 1818,45; found: 1818,$27 ;[\mathrm{M}+2 \mathrm{H}]^{2+}=910.14 ;[\mathrm{M}+3 \mathrm{H}]^{3+}=607.09$; r.t.:

$6.10 \mathrm{~min}$; yield: $40 \%$

n.10: TB_KKG6K(Ahx) Sequence: KKLLPIVK(Ahx)NLLKSLL

Calculated mass (Da):1832.15; found: $1832.44[\mathrm{M}+2 \mathrm{H}]^{2+}=917.22$; $[\mathrm{M}+3 \mathrm{H}]^{3+}=612.16$; r.t.: 10.9 min; yield: $45 \%$. 
n.11: TB_KKG6K(BrHx) Sequence: KKLLPIVK(BrHx)NLLKSLL

Calculated mass (Da):1897,89; found: $1898,34[\mathrm{M}+2 \mathrm{H}]^{2+}=950.17 ;[\mathrm{M}+3 \mathrm{H}]^{3+}=633.79$; r.t.:

$6.40 \mathrm{~min}$; yield: $40 \%$

n.12: TB_KKG6K(Aund) Sequence: KKLLPIVK(Aund)NLLKSLL

Calculated mass (Da): 1901.54; found: $1902.37[\mathrm{M}+2 \mathrm{H}]^{2+}=952.19$; $[\mathrm{M}+3 \mathrm{H}]^{3+}=635.13$. r.t.:

9.2 min; yield: $30 \%$.

\subsection{Critical aggregation concentration}

The critical aggregation concentration was determined for the peptide TB_KKG6K(Pal) and TB_KKG6K by fluorescence on a Jasco Cary eclipse instrument, setting up the excitation wavelength at $350 \mathrm{~nm}$. Increasing concentrations of peptide were titrated into a $20 \mu \mathrm{M}$ 8-amino naphtalen sulfonic acid solution in water. Emission at $470 \mathrm{~nm}$ was plotted against peptide concentration. The critical aggregation concentration was determined at the crossing point of the two lines at different slope obtained fitting by a linear regression fluorescence intensity before and after aggregation versus the concentration of the peptide.

\subsection{Killing kinetics determination}

Killing kinetics were investigated for the peptides on two different bacteria strains: E.coli and S.epidermidis. Bacteria were grown on Tryptic Soy Agar Petri dishes at $37^{\circ} \mathrm{C}$ over night before the treatment. The day of treatment a bacterial smear was added to $2 \mathrm{ml}$ of LB (Luria-Bertani liquid medium) to obtain $0.5 \mathrm{McF}$ Frland units turbidity, corresponding to about $1 \times 10^{8} \mathrm{CFU} / \mathrm{ml}$ bacterial concentration. ${ }^{[18]} \mathrm{A} 1.3 \mu \mathrm{l}$ volume of this bacterial suspension were inoculated in $2 \mathrm{~mL}$ of LB medium for each test sample to obtain a final bacterial concentration of $6.6 \times 10^{4} \mathrm{CFU} / \mathrm{ml}$. Gentamicin was used as positive control at the concentration of $1.5 \mu \mathrm{g} / \mathrm{ml}$ for E.coli and $1 \mu \mathrm{g} / \mathrm{ml}$ for S.epidermidis. The treatment was carried out at two different concentrations (50 $\mu \mathrm{M}$ and $10 \mu \mathrm{M})$ of each peptide. The test tubes were incubated at $37^{\circ} \mathrm{C}$ in shake for 24 hours. The turbidity was measured every two hours as proposed by Sutton (2011), using Densitometer DEN-1B (Grant bio). ${ }^{[19]}$

\subsection{Minimum Inhibitory Concentration (MIC) determination}

The MIC assays for the peptides were carried out in triplicate on three bacteria strains (E.coli, S.epidermidis and P.aeruginosa) by using the microdilution method. Bacteria were grown on Tryptic Soy Agar Petri dishes at $37^{\circ} \mathrm{C}$ over night. The day of treatment a bacterial smear was added to $2 \mathrm{ml}$ of LB or TSB (Tryptic Soy Broth) for E.coli and S.epidermidis or P.aeruginosa respectively, to obtain 0.5 McFarland units turbidity (about $\left.1 \times 10^{8} \mathrm{CFU} / \mathrm{ml}\right){ }^{[18]}$ Different concentrations of each peptide were added to $2 \mathrm{ml}$ of corresponding strain medium inoculated with $1.3 \mu \mathrm{l}$ of bacterial suspension to obtain a final concentration of $6.6 \times 10^{4} \mathrm{CFU} / \mathrm{ml}$, followed by incubation for up to 8 hours at $37^{\circ} \mathrm{C}$ in shake. Gentamicin was used as positive control. Bacterial growth was detected by turbidity 
measurement using Densitometer DEN-1B (Grant bio). MIC was defined as the lowest peptide concentration that inhibited bacterial growth. ${ }^{[19]}$

\subsection{Cell cultures for CD experiments}

E.coli BL21 (DE3) and S.epidermidis (ATCC 12228) cells for CD experiments were grown in $\mathrm{LB}$ medium at $37^{\circ} \mathrm{C}$, harvested while in exponential phase $\left(O D_{600} \mathrm{~nm}: 0.6-0.8\right)$ and centrifuged (5000 rpm, $10 \mathrm{~min}, 4^{\circ} \mathrm{C}$ ). S.epidermidis cells were suspended in $\mathrm{Na}_{2} \mathrm{HPO}_{4} 10$ $\mathrm{mM}, \mathrm{KH}_{2} \mathrm{PO}_{4} 2 \mathrm{mM}, \mathrm{NaCl} 20 \mathrm{mM}, \mathrm{KCl} 2.7 \mathrm{mM}, \mathrm{pH}$, centrifuged three times and then resuspended in the same buffer at $0.1 \mathrm{OD}_{600}$. E.coli cells were suspended in $10 \mathrm{mM}$ phosphate buffer $\mathrm{pH} \mathrm{7,} \mathrm{centrifuged} \mathrm{three} \mathrm{times} \mathrm{and} \mathrm{then} \mathrm{re-suspended} \mathrm{in} \mathrm{the} \mathrm{same} \mathrm{buffer}$ at $0.1 \mathrm{OD}_{600 .}{ }^{[11,15 b]}$

\subsection{CD studies}

Spectra were recorded on a Jasco $\mathrm{J} 715$ spectrometer at $25^{\circ} \mathrm{C}$ using a $0.1 \mathrm{~cm}$ quartz cell in the range $260-195 \mathrm{~nm}$ setting the scanning speed at $200 \mathrm{~nm} / \mathrm{min}$, bandwidth $1 \mathrm{~nm}$, response time $2 \mathrm{~s}$, data pitch $1 \mathrm{~nm}$. Peptides concentration for CD measurement was 60 $\mu \mathrm{M}$; measurements were performed in : 1) $10 \mathrm{mM}$ sodium phosphate buffer $\mathrm{pH} 7.0$ 2) $\mathrm{Na}_{2} \mathrm{HPO}_{4} 10 \mathrm{mM}, \mathrm{KH}_{2} \mathrm{PO}_{4} 2 \mathrm{mM}, \mathrm{NaCl} 20 \mathrm{mM}, \mathrm{KCl} 2.7 \mathrm{mM}$ at pH 7; 3) E.coli cells 0.1 $\mathrm{OD}_{600}$ in $10 \mathrm{mM}$ sodium phosphate buffer $\mathrm{pH} 7.0$ 4) S.epidermidis cells $0.1 \mathrm{OD}_{600}$ $\mathrm{Na}_{2} \mathrm{HPO}_{4} 10 \mathrm{mM}, \mathrm{KH}_{2} \mathrm{PO}_{4} 2 \mathrm{mM}, \mathrm{NaCl} 20 \mathrm{mM}, \mathrm{KCl} 2.7 \mathrm{mM}$ at pH 7. Peptide concentration was determined measuring the absorbance at $205 \mathrm{~nm}$ a Thermo Fisher Scientific Inc (Wilmington, Delaware USA) Nanodrop 2000c spectrophotometer.

Peptides were mixed to the bacterial cells; spectra were recorded every 20 minutes for 2 hours.

This research did not receive any specific grant from funding agencies in the public, commercial, or not-for-profit sectors.

\section{References}

[1] A. Majerle, J. Kidric, R. Jerala, J Antimicrob Chemother 2003, 51, 1159-1165.

[2] G. Laverty, S. P. Gorman, B. F. Gilmore, Chem Biol Drug Des 2015, 85, 645-652.

[3] J. J. Koh, H. Lin, V. Caroline, Y. S. Chew, L. M. Pang, T. T. Aung, J. Li, R. Lakshminarayanan, D. T. Tan, C. Verma, A. L. Tan, R. W. Beuerman, S. Liu, J Med Chem 2015, 58, 6533-6548.

[4] D. Avrahami, Y. Shai, J Biol Chem 2004, 279, 12277-12285.

[5] H. Heerklotz, J. Seelig, Biophys J 2001, 81, 1547-1554.

[6] R. Capparelli, A. Romanelli, M. Iannaccone, N. Nocerino, R. Ripa, S. Pensato, C. Pedone, D. lannelli, PLoS One 2009, 4, e7191.

[7] a) C. Avitabile, F. Netti, G. Orefice, M. Palmieri, N. Nocerino, G. Malgieri, L. D. D'Andrea, R. Capparelli, R. Fattorusso, A. Romanelli, Biochim Biophys Acta 2013, 1830, 3767-3775; b) V. Bezzerri, C. Avitabile, M. C. Dechecchi, I. Lampronti, M. Borgatti, G. Montagner, G. Cabrini, R. Gambari, A. Romanelli, J Pept Sci 2014, 20, 822-830. 
[8] G. Malgieri, C. Avitabile, M. Palmieri, L. D. D'Andrea, C. Isernia, A. Romanelli, R. Fattorusso, ACS Chem Biol 2015, 10, 965-969.

[9] S. Pellegrino, C. Annoni, A. Contini, F. Clerici, M. L. Gelmi, Amino Acids 2012, 43, 1995-2003.

[10] a) M. R. Yeaman, N. Y. Yount, Nat Rev Microbiol 2007, 5, 727-740; b) A. Bhunia, R. Saravanan, H. Mohanram, M. L. Mangoni, S. Bhattacharjya, J Biol Chem 2011, 286, 24394-24406.

[11] C. Avitabile, L. D. D'Andrea, M. Saviano, A. Romanelli, Rsc Adv 2016, 6, 51407-51410.

[12] C. Avitabile, L. D. D'Andrea, M. Saviano, M. Olivieri, A. Cimmino, A. Romanelli, Biochem Biophys Res Commun 2016, 478, 149-153.

[13] B. F. Lin, D. Missirlis, D. V. Krogstad, M. Tirrell, Biochemistry 2012, 51, 4658-4668.

[14] D. Missirlis, M. Farine, M. Kastantin, B. Ananthanarayanan, T. Neumann, M. Tirrell, Bioconjug Chem 2010, 21, 465-475.

[15] a) H. Mohanram, S. Bhattacharjya, Biochim Biophys Acta 2016, 1860, 1362-1372; b) C. Avitabile, L. D. D'Andrea, A. Romanelli, Sci Rep 2014, 4, 4293.

[16] S. Pellegrino, N. Ferri, N. Colombo, E. Cremona, A. Corsini, R. Fanelli, M. L. Gelmi, C. Cabrele, Bioorg Med Chem Lett 2009, 19, 6298-6302.

[17] M. Gaglione, G. Milano, A. Chambery, L. Moggio, A. Romanelli, A. Messere, Mol Biosyst 2011, 7, 2490-2499.

[18] F.C. Tenover in Encyclopedia of Microbiology, Third Edition (Ed.: Schaechter M.), Academic Press, Cambridge, 2009, pp 67-77.

[19] S. Sutton, Journal of Validation Technology, 2011, 17, 46-49. 